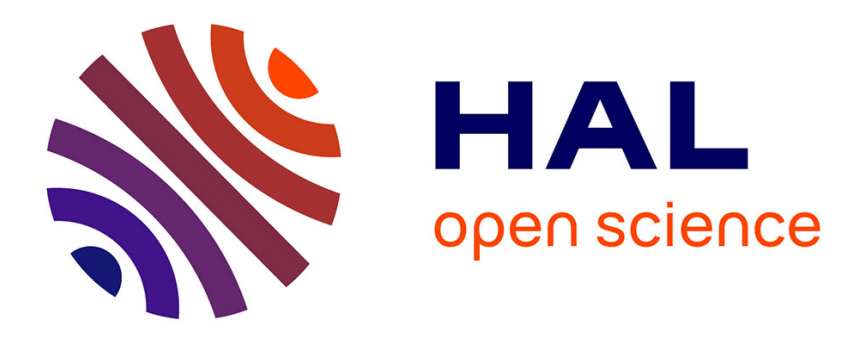

\title{
Une inscription tamoule inédite au musée d'Histoire du Vietnam de Hô Chi Minh-Ville
}

\author{
Emmanuel Francis
}

\section{To cite this version:}

Emmanuel Francis. Une inscription tamoule inédite au musée d'Histoire du Vietnam de Hô Chi Minh-Ville. Bulletin de l'Ecole française d'Extrême-Orient, 2008, 95-96, pp.406-423. 10.3406/befeo.2008.6115 . halshs-00861817

\section{HAL Id: halshs-00861817 https://shs.hal.science/halshs-00861817}

Submitted on 19 Apr 2020

HAL is a multi-disciplinary open access archive for the deposit and dissemination of scientific research documents, whether they are published or not. The documents may come from teaching and research institutions in France or abroad, or from public or private research centers.
L'archive ouverte pluridisciplinaire HAL, est destinée au dépôt et à la diffusion de documents scientifiques de niveau recherche, publiés ou non, émanant des établissements d'enseignement et de recherche français ou étrangers, des laboratoires publics ou privés. 


\section{Une inscription tamoule inédite au musée d'Histoire du Vietnam} de Hô Chi Minh-Ville

\section{Emmanuel Francis}

\section{Citer ce document / Cite this document :}

Francis Emmanuel. Une inscription tamoule inédite au musée d'Histoire du Vietnam de Hô Chi Minh-Ville. In: Bulletin de l'Ecole française d'Extrême-Orient. Tome 95-96, 2008. pp. 409-424;

doi : https://doi.org/10.3406/befeo.2008.6115

https://www.persee.fr/doc/befeo_0336-1519_2008_num_95_1_6115

Fichier pdf généré le 08/11/2019 


\title{
Une inscription tamoule inédite au musée d'Histoire du Vietnam de Hô Chi Minh-Ville
}

\author{
Emmanuel Francis*
}

\begin{abstract}
En janvier 2007, j'ai appris grâce à Bertrand Porte l'existence d'une inscription tamoule inédite dont le texte se lit en continu sur deux pièces conservées dans la réserve du musée d'Histoire du Vietnam (Bảo tàng Lịch sử [BTLS]) de Hô Chi Minh-Ville. Gerdi Gerschheimer transmit l'ensemble des documents disponibles - photographies des objets par Bertrand Porte et des estampages de l'inscription réalisés par l'EFEO à une date non déterminée - à des collègues tamoulisants susceptibles de lire et de traduire cette inscription. Il s'ensuivit un échange de courriels entre indianistes et spécialistes de l'Asie du Sud-Est impliquant E. Edwards McKinnon, Dominic Goodall, Arlo Griffiths, Andrew Hardy, Amandine Lepoutre, Natasha Pairaudeau, Philippe Papin, Bertrand Porte, Charlotte Schmid, William Southworth, Dominique Soutif et Olivier Tessier. La présente chronique se veut l'édifice modeste que chacune des pierres amenées par ces différents intervenants a permis de construire. Qu'ils soient tous remerciés pour les lumières qu'ils ont apportées.
\end{abstract}

\section{Les pièces}

L'inscription est gravée sur deux blocs de pierre ${ }^{1}$ de forme parallélépipédique : le parallélépipède $\mathrm{n}^{\circ} 1$, portant probablement le numéro d'inventaire «BTLS 5854 », et le parallélépipède $\mathrm{n}^{\circ} 2$, portant le numéro d'inventaire «BTLS 5853 » (ill. 1 et 2). Ces deux pièces sont associées dans les réserves du musée d'Histoire du Vietnam à un troisième bloc dont la forme en arc brisé évoque celle d'un tympan (numéro d'inventaire «BTLS 5853 »; ill. 1). Nous verrons que cette pièce n'est peut-être pas un tympan, mais je la dénommerai comme telle par convention. Ce tympan $\left(\mathrm{n}^{\circ} 1\right)$ est sculpté d'un personnage à seize bras sur ce qui doit être sa face avant. L'autre face ne porte en effet qu'une ébauche en creux d'un personnage très semblable. Il existe un deuxième tympan (probable numéro d'inventaire «BTLS $5854 »$; ill. 5) portant lui aussi la représentation d'un même personnage. Cependant, sa position - à l'exception des pieds - et ses attributs sont inversés par rapport au personnage du tympan $\mathrm{n}^{\circ} 1$. Ce tympan $\left(\mathrm{n}^{\circ} 2\right)$ appartient également à la collection du musée d'Histoire du Vietnam et était exposé en 2007 au musée des Beaux-Arts (Bảo tàng Mỹ thuật) de Hô Chi Minh-Ville où il avait été transféré. Il y est encore conservé, mais je n'ai pu établir si sa face arrière comporte, elle aussi, une représentation. Ce deuxième tympan a été publié et sévèrement jugé d'un point de vue esthétique par Jean Boisselier (1963, p. 394 et fig. 249). Manifestement ces quatre

* Membre associé du Centre d'études de l'Inde et de l'Asie du Sud (CNRS-EHESS).

1. Il s'agit d'une roche blanchâtre-rosée aux stries très marquées, peut-être un grès. 
objets - les deux parallélépipèdes et les deux tympans - aujourd'hui dispersés dans deux musées forment un ensemble? .

Dominique Soutif m'a signalé que, d'après une note manuscrite les accompagnant, les estampages EFEO de cette inscription (n. 1187; ill. 3 et 4 ) furent réalisés alors que la pierre était « conservée dans le temple funéraire de Do-hun-phuong ». Mais il faut corriger cette information en se fiant à $\mathrm{J}$. Boisselier (1963, ad fig. 249), qui décrit le tympan $\mathrm{n}^{\circ} 2$ comme « provenant du Tombeau du Tông-đôc Đò-hửu Phương ». Tổng đốc est un titre de fonctionnaire qui désigne un gouverneur de région ou de province. D'après des informations disponibles sur internet, Đỗ Hữu Phương (1841-1914) aurait servi dans l'administration coloniale française et aurait même été naturalisé français ${ }^{3}$. Il aurait fondé en 1904 un temple funéraire et familial, connu sous le nom de « đền Bà Lớn » (《le temple de la grande dame », en référence à son épouse) et situé au n ${ }^{\circ} 611 \mathrm{C}$ de la rue Dien Bien Phu à Saigon ${ }^{4}$.

\section{L'inscription}

L'inscription comporte huit lignes. Elle se répartit à raison de quatre lignes par parallélépipède. Aux extrémités des faces inscrites sont figurés des petits personnages levant les deux bras en fonction d'atlantes. L'inscription commence sur le parallélépipède supérieur $\left(n^{\circ} 1\right)$ et se poursuit sur le parallélépipède inférieur $\left(n^{\circ} 2\right)$ de l'illustration 1 . Le texte est en langue tamoule et en alphabet tamoul à l'exception d'un caractère en écriture grantha, $j a$, qui apparaît deux fois à la ligne 1. La gravure n'est pas particulièrement soignée puisqu'on observe une variation dans la taille des caractères et un alignement irrégulier. L'inscription n'est pas pour autant négligemment inscrite. Sur les photographies et estampages disponibles, elle est tout à fait lisible à l'exception de quelques caractères. La présence de pull i (point suscrit indiquant qu'une consonne n'est pas vocalisée) n'est pas toujours aisément déterminable et les deux derniers caractères de la dernière ligne sont de lecture incertaine.

L'examen de la paléographie permet de déterminer que cette inscription est de date relativement récente. Les caractères spécifiques utilisés pour noter le $\bar{o}$ et le $\bar{e}$ sont une invention revendiquée par Constanzo Gioseffo Eusebio (alias Constantius Joseph) Beschi dans la première moitié du $\mathrm{XVIII}^{\mathrm{e}}$ siècle $^{5}$. L'usage du pulli est rarement attesté

2. Une notice du musée d'Histoire du Vietnam décrit sous le numéro d'inventaire «BTLS 5853 » un des parallélépipèdes et un des tympans. Le numéro 5853 est d'ailleurs inscrit sur le parallélépipède inférieur (parallélépipède $n^{\circ} 2$, ill. 1 et 3 ) qui porte la deuxième moitié de l'inscription ainsi que sur une étiquette collée sur le tympan $n^{\circ} 1$. L'autre parallélépipède et l'autre tympan sont probablement regroupés sous le numéro d'inventaire «BTLS $5854 »$.

3. http://caonienbachhac.blogspot.com/2010/08/o-huu-phuong.html.

4. http://tintuc.xalo.vn/00-16046925/Ngoi_tu_duong_tram_nam_tuoi_bong_dung_bi_thu_hoi.html.

5. Eugen Hultzsch (1890, p. v-vi) précise que les caractères distinctifs pour ces deux voyelles $\bar{o}$ et $\bar{e}$ furent inventés par Beschi (1680-1747, selon Kamil Veith Zvelebil 1995, p. 92). E. Hultzsch renvoie à la Grammaire française-tamoule de Beschi (Pondichéry, 1863, p. 5) et à A. C. Burnell (1878, p. 45, note 4). Voir aussi A. C. Burnell 1874, p. 37 et note 4. Ce dernier cite un extrait de la Grammatica latino-tamoulica du kotuntamil de Beschi, qu'il date de 1739. Selon K. V. Zvelebil (1995, p. 94), cet ouvrage date de 1738 tandis que Stuart H. Blackburn (2003, p. 61) considère qu'il date plus probablement de 1728. Beschi (1997, p. 7-8) explique qu'un usage peu fréquent est de marquer e et $o$ d'un macron tandis que $\bar{e}$ et $\bar{o}$ n'ont pas de signes distinctifs, mais qu'il a inventé une convention que beaucoup de ses contemporains adoptèrent : $\bar{e}$ et $\bar{o}$ sont précédés d'un kompu recourbé dans sa partie supérieure (\&) tandis que e et o sont précédés du kompu normal (ด). 
à date ancienne dans l'épigraphie tamoule. On notera également la forme récente des chiffres ${ }^{6}$. Dominic Goodall relève en outre la ligature spécifique du «tt » dans cuttinatu à la ligne 4, qu'il n'a rencontrée que dans les portions tamoules qui suivent les colophons de manuscrits sanskrits de la collection de l'Institut français de Pondichéry, tous relativement récents ${ }^{7}$. Il semble donc difficile de considérer que notre inscription est antérieure au $\mathrm{XIX}^{\mathfrak{c}}$ siècle. Elle pourrait même dater du début du $\mathrm{XX}^{\mathrm{e}}$ siècle si l'ensemble des pièces fut exécuté pour le temple funéraire du Tổng đốc Đỗ Hữu Phương, décédé en 1914. Mais on ne peut exclure pour le moment que l'ensemble des pièces fut rapporté dans ce temple. L'inscription contient une date interne, mais elle n'aide guère à préciser la date de l'épigraphe car l'ère utilisée est incertaine.

Cette date est exprimée par quatre chiffres. Ces derniers sont suivis par un autre symbole ou une abréviation - un $a$ initial comportant un appendice du côté droit, que je note par@dans l'édition de l'inscription-qui signale manifestement que ces chiffres désignent un millésime. Le premier chiffre pose un problème : on peut lire en effet « 6 » ou « 9 ». Je penche, pour ce qui est inscrit, en faveur de « 6 », mais il peut s'agir d'une erreur, de la part de l'auteur ou du graveur, pour « $9 »$, tant on peut confondre facilement ces deux chiffres. Les trois chiffres suivants sont aisément reconnaissables : il s'agit de « $100 », 《 10 »$ et « 1 ». La date interne de l'inscription serait donc soit $6 \times 100+10+1$, à savoir 611 , soit $9 \times 100+10+1$, à savoir 911 .

L'ère utilisée n'est pas précisée. Dominic Goodall suggère une date en ère kali, pour laquelle il nous manquerait un chiffre au début, plus que probablement « $4 »^{8}$. S'il en est ainsi, l'année 4911 de l'ère kali qui correspond à l'année 1809/1810 de l'ère chrétienne est tout à fait recevable eu égard à la paléographie de l'inscription. Avec la lecture 4611 on obtient une date trop ancienne à ce même égard. Il est également possible qu'une ère locale ait été utilisée, mais l'usage au Vietnam était de dater en année de règne du souverain.

Du point de vue de l'orthographe et de la langue tamoules, les particularités suivantes trahissent des écarts, voire des fautes, par rapport à la norme : confusion dans la longueur des voyelles $(\bar{u}$ au lieu de $u$ dans vantù à la ligne 3 et dans irūntatu à la ligne $7 ; \bar{e}$ au lieu de $e$ dans $c \bar{e} y t u$ à la ligne 8); substitution de $r$ par $\underline{r}$ dans $\underline{r} \overline{a j a}$ à la ligne 1 . Dominic Goodall signale également que cuttinatu à la ligne 4 et cuttum à la ligne 5 relèvent de la langue parlée. La racine dont la forme littéraire est currutal (« faire tourner; tourner autour de ») est en effet utilisée de nos jours dans le sens de « se promener, faire du tourisme ». Cet usage appuie également une date relativement récente pour l'inscription. On notera aussi à la ligne 1 l'emploi côte à côte de $m a k \bar{a}^{\circ}$ et de sa variante $m \bar{a}^{\circ}$ dans ${ }^{\circ} m a k a \bar{r} a \bar{j} j a^{\circ}$ et 'mārajan, tous deux équivalents au sanskrit mahārāja.

Du point de vue grammatical, il est remarquable que toutes les formes verbales puissent s'analyser comme des formes non finies, participiales en l'occurrence. Seuls āki[n] atu à la ligne 6 et irüntatu à la ligne 7 peuvent éventuellement s'analyser comme des formes finies d'indicatif passé, mais le fait que cuttinatu à la ligne 4 ne puisse être analysé que

6. Ces chiffres tamouls ressemblent à des lettres comme les chiffres indiens anciens, dont ils semblent être une survivance. On considère généralement que ces chiffres anciens sont des lettres utilisées pour écrire des nombres (voir Georg Bühler 1987, p. 97-102, en particulier p. 101-102). Voir cependant les remarques judicieuses de Jean Filliozat (1953, p. 702).

7. Dominic Goodall précise que les manuscrits explicitement datés les plus anciens de l'Institut français de Pondichéry datent des années 1780 et que les plus récents, sur ôles, datent du tout début du xx siècle.

8. L'omission du premier chiffre du millésime est attestée au Cachemire. 
comme un nom participial passé au nominatif neutre singulier (car son sujet est masculin) incline à penser que les deux formes précitées le sont également.

Les inscriptions tamoules anciennes produites hors de l'Inde en Asie du Sud-Est et en Chine, c'est-à-dire en excluant les inscriptions en écriture tamil-brāhmī sur des objets peut-être exportés d'Inde, ne sont pas légion. Je n'en connais que sept provenant de Takua Pa (sud de la Thaïlande; milieu du I $X^{\mathfrak{C}}$ siècle), de Lobo Tua près de Barus (côte nord-ouest de Sumatra; 1010 śaka $=1088$ de n. è.), de Nakhon Si Thammarat (sud de la Thaïlande; 1183 ou 1283 śaka = 1261 ou 1361), de Porlak Dolok (nord de Sumatra, non loin de Barus, à l'intérieur des terres; 11[..] śaka $=1187,1258$ ou 1265 selon Noboru Karashima 2002, p. 14, d'après les détails astronomiques fournis), de Pagan (Birmanie; $\mathrm{XIII}^{\mathrm{e}}$ siècle), de Neusu à Aceh (extrémité nord de Sumatra; $\mathrm{XII}^{\mathrm{e}}$ siècle selon Jan Wisseman Christie 1998, p. 258; $\mathrm{XIII}^{\mathrm{e}}$ siècle selon Y. Subbarayalu 2009, p. 529) et de Quanzhou (côte sud de la Chine; 1203 śaka = 1281).

J. W. Christie (1998) recense huit inscriptions mais compte erronément (p. 262) une inscription du roi Âdityavarman (deuxième moitié du XIII ${ }^{\mathbb{e}}$ siècle) provenant de Batu Bapahat, sur la côte ouest de Sumatra. Deux inscriptions de ce roi, aujourd'hui disparues, proviennent en fait de ce site. La première est en sanskrit et en écriture kawi. La seconde, très lacunaire, est en écriture grantha du sud de l'Inde et apparemment également en sanskrit. Il apparaît que plusieurs chercheurs ont mal interprété les rapports concernant cette inscription inédite et ont conclu qu'elle était composée en tamoul à cause du type d'écriture utilisée. J'ai pu constater, avec Arlo Griffiths en octobre 2009 lors de l'examen de l'estampage non encré de cette inscription, que ses parties lisibles sont effectivement en sanskrit. Cet estampage conservé dans les collections spéciales de la bibliothèque de l'université de Leyde et une photographie (numéro d'inventaire «OD 1646 ») du service archéologique des Indes néerlandaises (Oudheidkundige Dienst in Nederlandsch-Indië) ${ }^{9}$ sont, à notre connaissance, les seuls documents subsistant pour lire cette inscription.

Noboru Karashima (2002) recense de son côté sept inscriptions. Il n'inclut pas - à raison - l'inscription de Batu Bapahat, mais omet celle de Neusu connue de J. W. Christie (1998) et éditée par Y. Subbarayalu (2009). Il inclut en outre (p. 11) une inscription en écriture tamil-brāhmī (III ${ }^{\mathrm{e}}-\mathrm{IV}^{\mathrm{c}}$ siècles) gravée sur une pierre de touche découverte dans le sud de la Thaïlande à Khuan Luk Pat ${ }^{10}$. 11 est possible cependant que cet objet de petite dimension ait été amené d'Inde.

Le seul fait que notre inscription soit en tamoul lui confère donc un certain intérêt, eu égard au nombre restreint d'inscriptions tamoules produites hors de l'Inde. Néanmoins sa date récente n'en fait pas un document de première importance pour l'histoire ancienne des contacts entre l'Inde et l'Asie du Sud-Est.

9. La collection de plus de vingt mille photographies du service archéologique des Indes néerlandaises déposées dans des collections publiques néerlandaises est intégrée dans une base de données consultable sur https://socrates.leidenuniv.nl. Voir la collection « Kern Institute». Pour la recherche des documents, préfixer «OD- »- avec le tiret ! - au numéro demandé. Voir « OD 1645 » pour l'inscription sanskrite en écriture kawi d'Ādityavarman.

10. Sur cette inscription, voir aussi Jan Wisseman Christie (1998, p. 247, n. 31 ). 


\section{Texte de l'inscription ${ }^{11}$}

Parallélépipède $\mathrm{n}^{\circ} 1$ :
(1) alr]ccantaramakārājamārājan
(2)19]100101@annāam.tē-
(3) cattil. vantū kātu-
(4) ccām.tècam. cuttinatu

Parallélépipède $\mathrm{n}^{\circ} 2$ :

(5) kātu cuttum pōtuy i-

(6) ntay itam äki[ña]tu

(7) kōyil ākay irūntatu

(8) pūcai cèeytu kāninikkai]

L. 2 : [9]. La lecture « 6 » est possible.

L. 2 :@. L'arobase transcrit un $a$ initial comportant un appendice du côté droit. Ce symbole ou abréviation peut se comprendre soit comme -ām ou -āvatu («-ième », morphème qui transforme un numéral cardinal en numéral ordinal), comme me l'a suggéré Dominic Goodall, soit comme une abréviation pour ăntu, " année ». On attendrait cependant un « $\overline{\mathrm{a}} »$ comme premier membre du symbole. Arlo Griffiths suggère également qu'il pourrait s'agir de l'abréviation de $a[\dot{n} k a]$, utilisée en Orissa pour dénoter les années de règne des rois Gajapati, bien que la lettre y précède toujours les chiffres.

L. 3 : vantū. Lire vantu.

L. 4 : cuttinatu. La ligature tti porte un pulli qui n'est pas nécessaire.

L. $6: \bar{a} k i[\underline{n} a] t u$. Le caractère $n a$ est douteux à cause de l'état de la pierre. On peut proposer également la ou $r a$, mais n na est le plus vraisemblable : áki[la]tu ne ferait pas sens et $\bar{a} k i[\underline{r} a] t u$ serait la seule forme verbale au présent de toute l'inscription.

L. 7 : irūntatu. Lire iruntatu.

L. 8 : cèytu. Lire ceytu.

L. 8 : kāni [kkai]]. On peut également lire kāni [kal], kāni [kan]] ou kāni [can]]. Dominic Goodall lit kāni [k]kai sans exclure kānilkan] ou kāni[can]. Je lis pour ma part kānini[kal] ( « terre; propriété foncière »), mais j'admets qu'il semble y avoir un pulli au-dessus de l'avant-dernier caractère $k$, ce qui confirme la première lecture kānikkai. En outre kāṇi est habituellement utilisé au singulier. Il semble donc que le dernier caractère kai a été mal gravé par manque de place.

\section{Traduction}

"Arccantaran, grand roi parmi les grands rois, en l'an $911^{12}$ vint dans le pays d'Annam (ann̄ām) (et) visita le pays de jungle des Cam (cām) (ou : le pays des Cam de jungle). Tandis qu'il se promenait dans la jungle, il arriva en cet endroit ${ }^{13}$. Il se trouvait qu'il y

11. Les caractères grantha sont en romain, les lectures conjecturales entre crochets. Le point médian (« • ") à la fin d'un mol indique que le caractère final de ce mot est surmonté d'un pulli. Les espaces non gravés dans l'original sont reproduits dans la translittération.

12. Ou : « en l'an $611 »$, « en la $911^{\mathrm{c}}$ [année] », « en la $611^{\mathrm{c}}$ [année] ».

13. Littéralement : « cet endroit est advenu ». 
avait un temple ${ }^{14}$. Après avoir fait une pijjā («adoration, culte »), (il y fit) des offrandes $(\text { kānikkai })^{15} .{ }^{\prime}$

\section{Commentaires}

La personne dont l'inscription commémore les actes pieux se définit comme « grand roi parmi les grands rois » (makārājjamāărājan, équivalent du sanskrit mahārājamahārāja) et se nomme Arccantaran. Ce nom est vraisemblablement le calque tamoul approximatif d'un nom sanskrit : Haracandra pour lequel la forme attendue serait Araccantiran ou encore, comme me le suggère Dominic Goodall, Haricandra ou Hariścandra. Arccantaran n'est pas connu par ailleurs. Il est probable que sa désignation comme " grand roi parmi les grands rois » soit pompeuse. Il s'agit peut-être d'un voyageur tamoul qui se serait aventuré dans l'actuel Vietnam pour une raison obscure. À moins, comme le suggère Bruno Dagens, qu'il soit un fonctionnaire originaire des comptoirs français en pays tamoul, éduqué au Lycée français de Pondichéry ${ }^{16}$. En effet, dès le milieu des années 1860 , l'administration coloniale en Cochinchine enrôla notamment des Tamouls originaires de Pondichéry et de Karikal pour répondre à ses besoins en fonctionnaires francophones (Natasha Pairaudeau 2009, p. 87).

Une autre possibilité, suggérée par E. Edwards McKinnon, consiste à considérer qu'Arccantaran n'est pas un personnage contemporain de l'inscription, mais une figure historique ou même légendaire dont on commémorerait la gloire passée ou imaginée. N. Pairaudeau $(2009$, p. 268-275) explique en effet que l'élite tamoule de Saigon, depuis les années 1920 au moins, se passionna pour le rôle de l'Inde dans l'histoire ancienne de l'Indochine et souscrivit notamment au concept de "Greater India ». N. Pairaudeau m'informe en outre que le nom Arccantaran ne correspond pas aux types de noms connus pour les immigrés indiens d'Indochine de la fin du XIX ${ }^{\mathrm{e}}$ siècle ${ }^{17}$.

Le terme Annam désigne à partir du début du $\mathrm{XIX}^{\mathrm{e}}$ siècle la réunion des seigneuries Trịnh et Nguyễn qui composaient ce que l'on appelait plus communément le Đại Việt, comme le confirme Philippe Papin. Bien qu'on puisse interpréter le texte comme signifiant que le pays des Cam fut visité après le Đại Việt et était donc indépendant de ce dernier, on peut également comprendre que le Campā était déjà ici une province du Đại Việt ${ }^{18}$.

La désignation kātuccām têcam peut se comprendre comme «le pays de jungle des Cam », c'est-à-dire littéralement « le pays des Cam (ou : le pays cam) [qui est] une jungle ». Mais peut-être faut-il comprendre, comme me le suggère Arlo Griffiths, « le pays des Cam de jungle ». Il pourrait alors s'agir de l'une des populations montagnardes longtemps englobées sous le terme générique - et péjoratif - Moi (« sauvages » ou «barbares des hautes terres ») par les Vietnamiens puis par la tradition coloniale française, et

14. Ou : « il se trouva (quelque bâtiment) comme ( $\bar{a} k a)$ un temple »?

15. Ou : «Afin qu'il y ait un temple ( $k \overline{o y} y i l a \bar{k} a)$, ce qu'il advint (irtūntatu), après avoir fait une püjāa: (ses) offrandes (kānikkai). Ou encore : « (il donna) après avoir fait une püjā des droits de propriété (kānnikal) », c'est-à-dire littéralement « ce qu'il advint après avoir fait une püjja : des droits de propriété ».

16. Le lycée de Pondichéry fut fondé en 1826 sous le nom de Collège royal. Il fut dénommé par la suite Collège colonial.

17. Sont attestés seulement des noms typiquement tamouls, musulmans, chrétiens et francisés.

18. Sur l'absorption du Campā par le Đại Việt à la croisée du XvIII et du XIX ${ }^{e}$ siècle, voir Po Dharma (1987, p. 72-74). 
plus particulièrement de l'une de celles parlant une langue austronésienne. On notera qu'il existe aujourd'hui parmi ces dernières une minorité ethnique, de langue dite chamique selon les classifications linguistiques contemporaines, dont l'appellation est Ra Glai, soit littéralement « gens-jungle » en cam. L'expression tamoule kätuccām técam pourrait en être une transposition directe.

Le sens précis des deux dernières lignes n'est pas clair. On peut comprendre, et ce semble le plus logique, que, lors de son périple, Arccantaran aboutit devant un temple et y fit une $p \bar{u} j \bar{a}$ et des offrandes (kânikkai). À moins qu'il ne faille comprendre qu'après une püjā il fit des dons de terres (kānikal). On peut même se demander si ce n'est pas Arccantaran lui-même qui établit le temple et y fit ensuite preuve de sa dévotion. L'inscription n'établit en tout cas pas de connexion entre Arccantaran et Đỗ Hữu Phương, dans le temple funéraire duquel ces pièces furent trouvées. On ignore donc si ces dernières furent exécutées pour parer ce temple ou bien si elles y furent rapportées. Le fait que le temple doté par Arccantaran se trouvait, selon le texte de l'inscription, dans la jungle, incline à penser que le tombeau de Đỗ Hữu Phương n'est pas le site originel de nos pièces. Cependant, si Arccantaran est une figure légendaire, ce tombeau fut peutêtre paré par des résidents tamouls de la Saigon coloniale. Des liens spécifiques entre la communauté tamoule et Đỗ Hữu Phương ne sont pas connus, mais pas impossibles. N. Pairaudeau $(2009$, p. 246) signale par exemple que le mausolée du général Lê Văn Duyệt (1764-1832) fut rénové grâce à des dons de commerçants tamouls à la fin des années $1930^{19}$.

\section{Les tympans}

On peut aussi s'interroger sur le rapport entre les deux parallélépipèdes inscrits et les deux tympans. Le fait qu'un des deux parallélépipèdes et un des deux tympans soient enregistrés sous un même numéro d'inventaire au musée d'Histoire du Vietnam confirme que ces quatre objets forment un ensemble.

Ils se répartissent manifestement en deux groupes formés chacun d'un parallélépipède et d'un tympan. Bertrand Porte a observé en effet la présence d'un tenon sur le tympan du musée d'Histoire du Vietnam $\left(n^{\circ} 1\right)$ et d'une mortaise sur chacun des parallélépipèdes ${ }^{20}$. Il apparaît ainsi que chaque tympan reposait sur un des parallélépipèdes faisant office non de linteau, mais de piédestal ou de base. Les atlantes qui encadrent les lignes d'écriture sur chacun des parallélépipèdes soutenaient les tympans. Il est dès lors vraisemblable que les deux couples formés d'un tympan et d'une base parallélépipédique étaient disposés sur le sol ou sur un élément architectural de part et d'autre de l'entrée du temple qu'ils paraient, soit en vis-à-vis avec les quatre premières lignes faisant face aux quatre dernières, soit côte à côte avec les quatre premières lignes à gauche de l'observateur et les quatre dernières à sa droite, se lisant ainsi en continuité. En tout cas, les personnages des tympans s'opposent de façon symétrique.

19. N. Pairaudeau note : « Tamil reverence for Lê Văn Duyệt was in keeping with that of Chinese migrants who propitiated the spirit of the general in the belief that in his death, as in his life, he continued to support their enterprises $"$.

20. La présence d'un tenon sur le tympan $n^{\circ} 2$ n'est pas confirmée, mais fort probable. 
Les tympans - même s'il ne s'agit probablement pas, comme nous venons de le voir, de tympans proprement dits - rappellent non seulement par leur forme, mais aussi par leur iconographie, d'authentiques tympans tels qu'ils sont communs dans l'art cam dès une période ancienne ${ }^{21}$. Deux pièces datées du $\mathrm{X}^{\mathrm{e}}$ siècle, provenant de Khương Mỹ et conservées au musée de Đà Nẵng mais de dimensions sensiblement plus grandes (ill. 6 et 7), constituent des parallèles remarquables ${ }^{22}$. Il s'agit de représentations de Śiva dansant à vingt-huit bras ${ }^{23}$. Les deux mains antérieures portent une vin̄ā en travers du buste. Plusieurs des mains postérieures tiennent un serpent. Le buste humain qui constitue la partie supérieure de ce serpent est visible en haut à gauche (pour l'observateur). Son corps de reptile est tenu dans les mains gauches du dieu. Enfin, sa queue apparaît tout en bas à droite (pour l'observateur) ${ }^{24}$. En outre, deux mains du dieu sont jointes au-dessus de la tête en un geste qui, selon J. Boisselier (1963, p. 184), « va devenir fréquent au Champa qui semble avoir été seul à l'utiliser dans toute l'Asie du Sud-Est ${ }^{25} »$.

J. Boisselier (1963, p. 394) avait déjà noté le parallèle entre ces deux sculptures de Khương Mỹ et celle du personnage d'un des deux tympans de Hô Chi Minh-Ville. Ce dernier n'a certes que seize bras, mais «l'objet indéfinissable » qu'il tient dans ses mains naturelles en travers du buste (un bâton peut-être) rappelle la vin̄a de Śiva, même s'il la tient un peu plus bas. On notera en outre que ses deux mains supérieures se joignent au-dessus de la tête comme deux des mains supérieures des Śiva de Khương Mỹ. Cependant elles ne sont pas simplement jointes, mais tiennent un objet marqué du signe du yin/yang. Pourrait-il s'agir d'une fleur (offerte en $p \bar{u} j \bar{a}$ ) ou d'un joyau ? Ce motif du yin/yang confirme la date récente de la sculpture, car son usage ne se serait répandu qu'au XIX ${ }^{\mathrm{e}}$ siècle parmi les communautés cam, comme me l'a signalé William Southworth ${ }^{26}$. Tous les autres bras de notre personnage tiennent ce qui ressemble à une corde. Du côté gauche, cette « corde » se termine par un cercle, et du côté droit par une forme plus ou moins sphérique qui semble avoir des yeux. Ce pourrait être un serpent stylisé.

Il semble donc que notre voyageur tamoul loua les services d'artistes locaux qui connaissaient leurs classiques, même si leur technique n'était plus aussi raffinée que

21. Voir par exemple les sculptures $\mathrm{n}^{\mathrm{os}} 104,107,122$ et 127, en forme d'arc brisé comme nos pièces, et les sculptures $n^{\circ} 61$ et 63 , en forme d'arc en plein-cintre, illustrées dans le catalogue du musée de sculpture de Đà Năng de Léon Vandermeersch \& Jean-Pierre Ducrest (1997). Toutes ces sculptures sont décrites comme des tympans et certaines ont été illustrées récemment dans le catalogue de l'exposition Trésors d'art du Vietnam. La sculpture du Champa. $v^{v^{\prime}}-x v^{\prime \prime}$ siècles (Pierre Baptiste \& Thierry Zéphir 2005).

22. Cf. J. Boisselier (1963, p. 184-185 et fig. 105-106), C. Sivaramamurti (1974, p. 352 et fig. 20-21). Pour la première sculpture, voir aussi L. Vandermeersch \& J.-P. Ducrest $\left(1997, \mathrm{n}^{\circ} 104\right.$, où figurent de façon erronée le numéro d'inventaire et la hauteur de la seconde sculpture); pour la seconde, voir aussi Nguyen Van Ku-Pham Ngoc Long \& Cao Xuan Pho (1988, n 173).

23. Pour des Śiva dansants du Cambodge, voir ceux de Baset et de Phnom Chisor que Bruno Dagens discute et illustre (1968, p. 183-184 et 191; fig. 9 et 17), les rapprochant notamment de ceux de Khương Mỹ.

24. Selon L. Vandermeersch \& J.-P. Ducrest (1997, p. 129), les « mains postérieures semblent tenir une corde ». Cette « corde » est à mon avis le corps du serpent. Pour des Śiva dansants tenant un serpent au-dessus de la tête, voir le corpus des Câlukya de Bādāmī.

25. Pour un geste similaire, voir L. Vandermeersch \& J.-P. Ducrest (1997, $\mathrm{n}^{\text {os }} 138$ et 141).

26. Voir Rie Nakamura (1999, p. 129, note 36) : «A symbol mark of yin-yang is found in various places in Cham villages, for ornamentation of houses, Bani's mosques, or ceremonial music instruments. I was told that the yin-yang symbol mark was introduced during the time of Nguyễn dynasty (18021885) when the Vietnamese court established an administrative system modelled after the Chinese Confucian system ». 
celle de leurs prédécesseurs, comme on peut en juger par l'aspect relativement grossier des sculptures des deux tympans ${ }^{27}$.

Il reste à discuter de l'identification et des attributs de ce personnage dont les seize bras indiquent le caractère surnaturel. Sur quoi marche-t-il? Sont-ce des vagues océanes ou, comme le suggère Charlotte Schmid, des montagnes? La corde qu'il semble tenir dans ses douze mains intermédiaires serait-elle effectivement un serpent? Et que tient-il dans ses deux mains inférieures? S'agit-il d'une lance? Est-ce un baraton? Ce personnage serait-il Arccantaran traversant la mer depuis son pays tamoul? S'agit-il d'un dieu, équipé d'un baraton et d'un serpent, qui vient de baratter l'océan et d'en retirer un joyau qu'il arbore fièrement au-dessus de la tête ${ }^{28}$ ? Peut-on en déduire que le temple où nos pièces figuraient était consacré à Vișṇu, le dieu maître d'œuvre du barattage de l'océan? Arccantaran serait-il représenté comme un dieu aux bras multiples barattant l'océan? Ou alors, étant donné qu'il y a deux personnages, faut-il les considérer comme les deux gardiens du sanctuaire, surpuissants en raison de leurs bras multiples, portant un bâton comme arme et tenant au-dessus de leur tête une fleur qu'ils apportent en hommage à la divinité qu'ils protègent? Autant de questions auxquelles je ne m'aventurerai pas à donner des réponses définitives et que je laisse à la libre appréciation des spécialistes du Vietnam qui voudraient s'intéresser à ces quatre pièces exposées à Hô Chi Minh-Ville.

\section{Remerciements}

Mesdames Tran Thi Thanh Dao et Tran Thi Phuy Phuong, respectivement conservatrice en chef et directrice du musée d'Histoire (Bảo tàng Lịch sử) du Vietnam de Hô Chi Minh-Ville, ont permis l'accès et la documentation des pièces conservées dans leur musée. Madame Lam Quang Thuy Nhien, conservatrice du musée des Beaux-Arts (Bảo tàng Mỹ thuật) de Hô Chi Minh-Ville, a transmis des informations concernant le tympan $\mathrm{n}^{\circ} 2$ conservé en dépôt dans son musée. Madame Tran Thuy Diem, conservatrice au musée de Đà Nẵng, m'a communiqué les dimensions et des photos des sculptures de Khương Mỹ conservées dans son musée. Vu Thi Mai Anh (centre EFEO de Hanoi) a bien voulu traduire du vietnamien en français la notice d'inventaire « BTLS 5853 ». Bruno Dagens a accepté de lire une première version de cette chronique et $\mathrm{m}$ 'a fait part d'utiles suggestions et remarques. Uthaya Veluppillai et $G$. Vijayavenugopal m'ont fait part de leurs commentaires sur le texte de l'inscription. Pierre Baptiste (musée Guimet) m'a permis de publier ses clichés de deux sculptures de Khương Mỹ. Last but not least, cette chronique n'existerait pas sans la diligence de Bertrand Porte, qui m'a transmis de nombreuses informations, précisions et commentaires ainsi que les résultats de ses propres enquêtes. À toutes et à tous j'exprime ici ma plus vive reconnaissance.

27. À propos du tympan no 2 , J. Boisselier (1963, p. 394) se refuse à « parler d'art » et évoque son « anatomie affligeante » ainsi que « l'impuissance des artisans » qui la confectionnèrent. Il concède cependant un double intérêt à cette sculpture qui « prouve tout à la fois la vitalité des traditions śivaïtes et l'influence du Việt-nam ».

28. Sur le baratlage de l'océan de lait par les Deva et les Asura qui utilisèrent le mont Mandara comme baraton et le serpent Vāsuki comme corde, voir Mahābhārata 1.15-17. Outre l'ambroisie (amrta), ce barattage produisit le joyau Kaustubha qui orne la poitrine de Viṣnu (Mahābhārata [.16.35). 


\section{Bibliographie}

BAPTISTE, Pierre \& Thierry ZéPhiR (dir.)

2005 Trésors d'art du Vietnam. La sculpture du Champa. $V^{e}-x V^{e}$ siècles, Paris, Réunion des musées nationaux / musée des Arts asiatiques-Guimet.

Beschi, Constantius Joseph

1997 A Grammar of the common Dialect of the Tamil Language called Kotuntamil. Translated from the original Latin by George William Mahoni, $2^{\mathrm{e}}$ éd., Tanjore, Sarasvati Mahal Library (Tanjore Sarasvati Mahal Series 133).

BI.ACKBURN, Stuart H.

2003 Print, Foklore and Nationalism in Colonial South India, Delhi, Permanent Black.

BoISSELIER, Jean

1963 La statuaire du Champa. Recherches sur les cultes et l'iconographie, Paris, EFEO (PEFEO 54).

BÜHLER, Georg

1987 Indian Paleography. From about B.C. 350 to about A.D. $1300,2^{\mathrm{c}}$ réimpr., Patna, Eastern Book House.

Burnell, A. C.

1874 Elements of South-Indian Palaeography. From the fourth to the seventeenth Century A.D. Being an Introduction to the Study of South-Indian Inscriptions

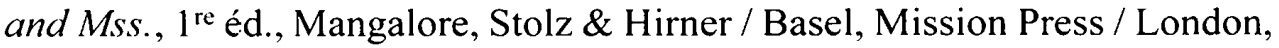
Trübner \& Co.

1878 Elements of South-Indian Palaeography. From the fourth to the seventeenth Century A.D. Being an Introduction to the Study of South-Indian Inscriptions and Mss., $2^{\mathrm{c}}$ éd., London, Trübner \& Co.

CHRISTIE, Jan Wisseman

1998 "The Medieval Tamil-Language Inscriptions in Southeast Asia and China », Journal of Southeast Asian Studies 29 (Sept. 1998), p. 239-268.

Dagens, Bruno

1968 «Étude iconographique de quelques fondations de l'époque de Sūryavarman I Ir », Arts asiatiques 17, p. 173-208.

Filliozat, Jean

1953 "Appendice 1. Paléographie », dans Louis Rinou \& Jean Filliozat, avec le concours de Paul Demit:ville, Olivier Lacombe et Pierre Meilf, L'Inde classique. Manuel des études indiennes, tome II, Paris, EFEO, p. 665-712.

Huitzsch, Eugen (éd. et trad.)

1890 South Indian Inscriptions, Tamil and Sanskrit, from Stone and Copper-Plate Edicts at Mamallapuram, Kanchipuram, in the North Arcot District, and other Parts of the Madras Presidency, chiefly collected in 1886-8, Vol. I, Madras, Government Press. 
Karashima, Noboru

2002 "Tamil Inscriptions in Southeast Asia and China ", dans Noboru KarAsHImA (éd.), Ancient and medieval commercial Activities in the Indian Ocean. Testimony of Inscriptions and Ceramic-sherds. Report of the Taisho University Research Project 1997-2000, Tokyo, Taisho University, p. 10-18.

Long, Nguyen Van Ku-Pham Ngoc (photographies) \& Cao Xuan Pho (texte) 1988 Cham Sculpture Album, Hanoi, Social Sciences Publishing House.

\section{Mahābhārata}

1933-59 The Mahābhārata. For the first time critically edited by Vishnu S. Sukthankar, S. K. Bevalkar, Parashuram Lakshman Vaidya [et al.], 19 vol., Poona, Bhandarkar Oriental Research Institute.

Nakamura, Rie

1999 «Cham in Vietnam: Dynamics of Ethnicity », PhD dissertation, University of Washington, Department of Anthropology.

PAiRAudeau, Natasha

2009 "Indians as French Citizens in Colonial Indochina, 1858-1940 ", PhD dissertation, University of London, School of Oriental and African Studies, Department of History.

Quang, Po Dharma

1987 Le Pānduranga (Campā) 1802-1835 et ses rapports avec le Vietnam, tome 1, Paris, EFEO (PEFEO 149).

Sivaramamurti, C.

1974 Națarajja in Art, Thought and Literature, New Delhi, National Museum.

SubBarayalu, Y.

2009 «A Trade Guild Tamil Inscription at Neusu, Aceh», dans Daniel Plerret \& Heddy Surachman (éd.), Histoire de Barus, Sumatra. III. Regards sur une place marchande de l'Océan Indien (XII'-milieu du XVIr's.), Paris, association Archipel (Cahiers d'Archipel 38), p. 529-532.

VANDERMEerscil, Léon \& Jean-Picrrc DuCREst (dir.)

1997 Le Musée de Sculpture Cam de Đà Nã̃ng, Paris, Association française des amis de l'Orient.

Zvelebil, Kamil Veith

1995 Lexicon of Tamil Literature, Leiden / New York / Köln, Brill (Handbuch der Orientalistik 2.9). 


\section{Illustrations}

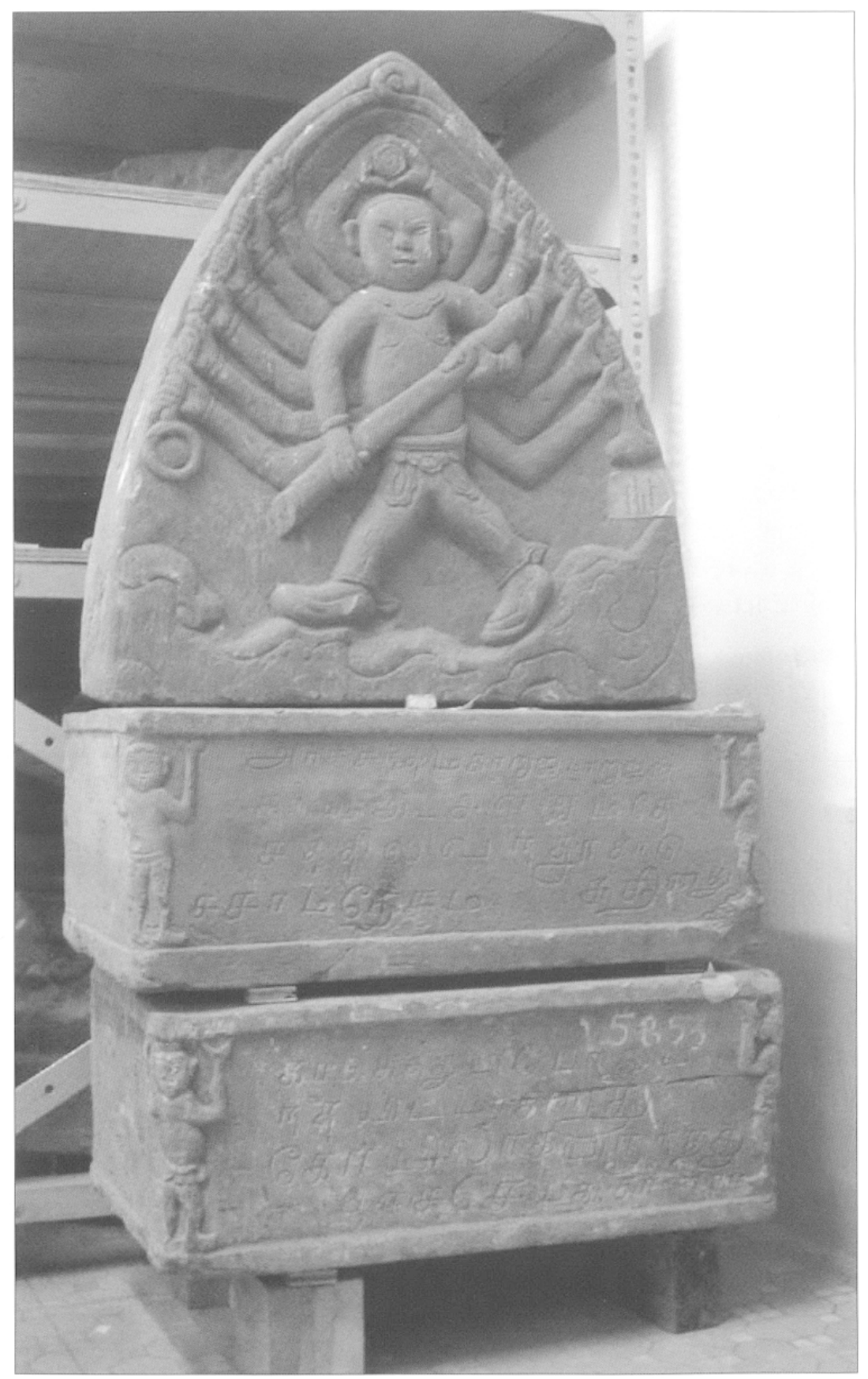

Ill. 1 : De haut en bas, tympan no 1 (numéro d'inventaire « BTLS 5853 »; hauteur : $75,5 \mathrm{~cm}$, dont $12,5 \mathrm{~cm}$ pour le tenon; largeur : $57 \mathrm{~cm}$; épaisseur : $11 \mathrm{~cm}$ ), parallélépipède n* 1 (numéro d'inventaire probable «BTLS 5854 »; hauteur : $23,5 \mathrm{~cm}$; largeur : $59 \mathrm{~cm}$; épaisseur : $32 \mathrm{~cm}$ ) et parallélépipède $\mathrm{n}^{\circ} 2$ (numéro d'inventaire «BTLS 5853 »; hauteur: $22,5 \mathrm{~cm}$; largeur : $58 \mathrm{~cm}$; épaisseur : $35 \mathrm{~cm}$ ), réserve du musée d'Histoire du Vietnam de Hô Chi Minh-Ville (cliché : Bertrand Porte, décembre 2006). 


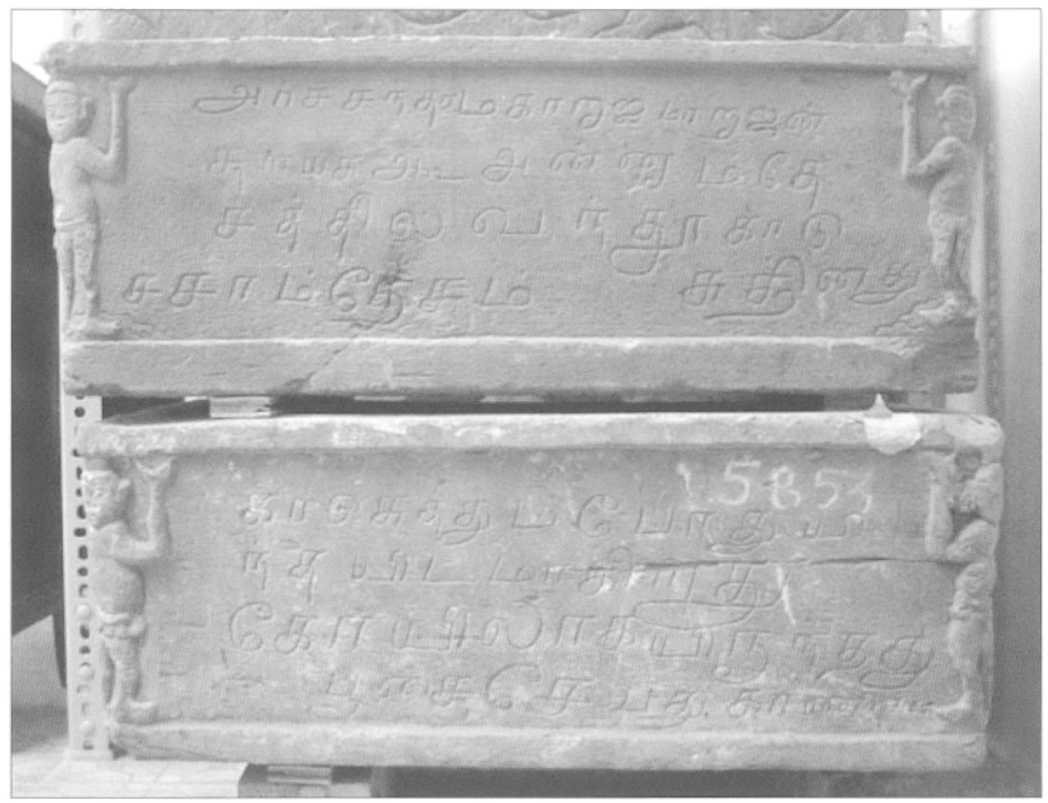

Ill. 2 : L'inscription (cliché : Bertrand Porte, décembre 2006).

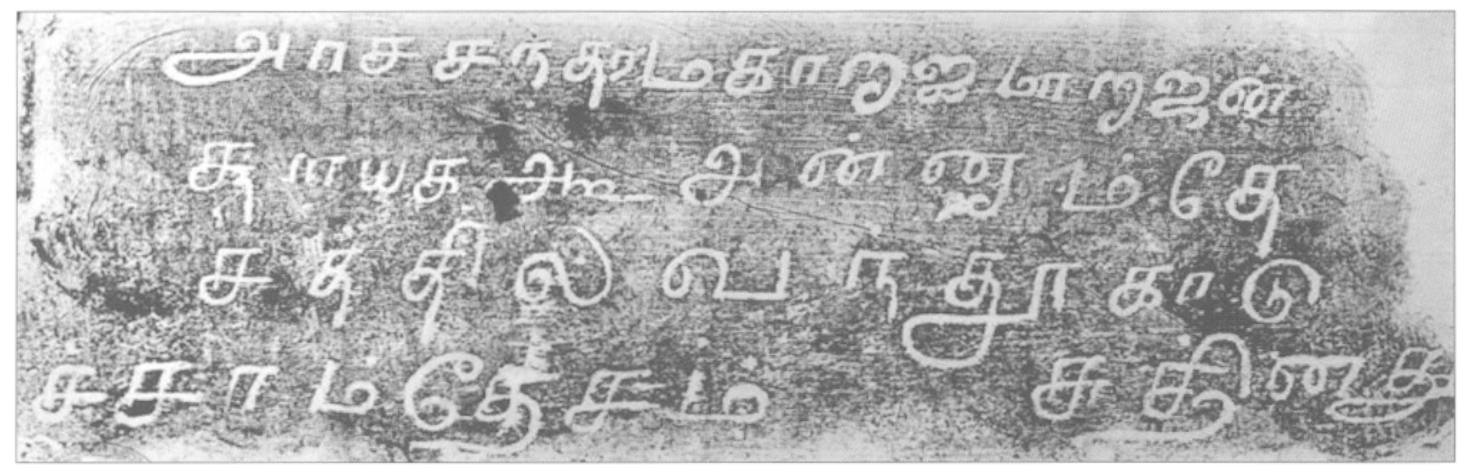

III. 3 : Lignes 1-4 de l'inscription (parallélépipède $n^{\circ}$ 1), estampage EFEO n. 1187.

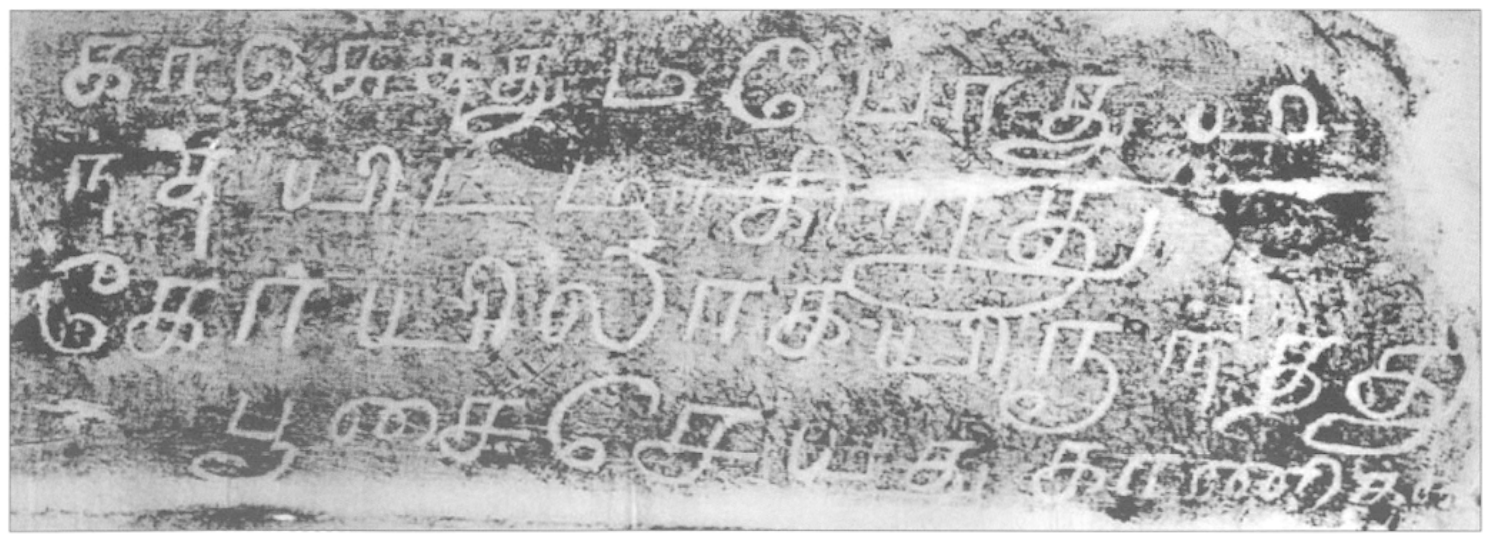

Ill. 4 : Lignes 5-8 de l'inscription (parallélépipède $n^{\circ} 2$ ), estampage EFEO n. 1187. 


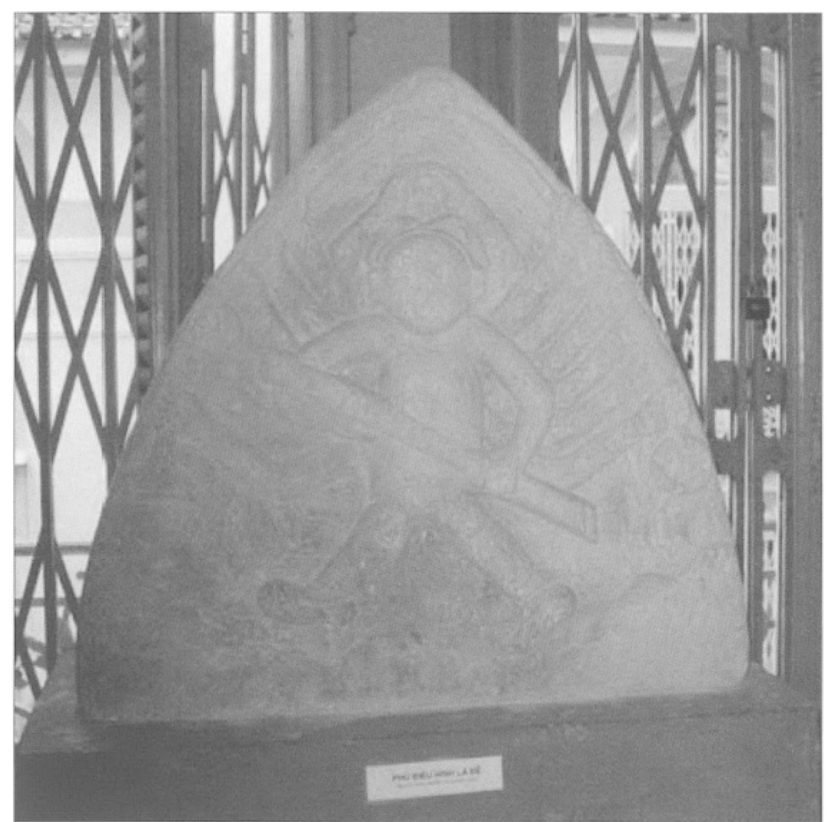

Ill. 5 : Tympan $n^{\circ} 2$, musée des Beaux-Arts de Hô Chi Minh-Ville (probable numéro d'inventaire : « BTLS 5854 "; dimensions exactes non connues, mais sensiblement similaires à celles du tympan $n^{\circ} 1$ ) (cliché : Bertrand Porte, juillet 2007).

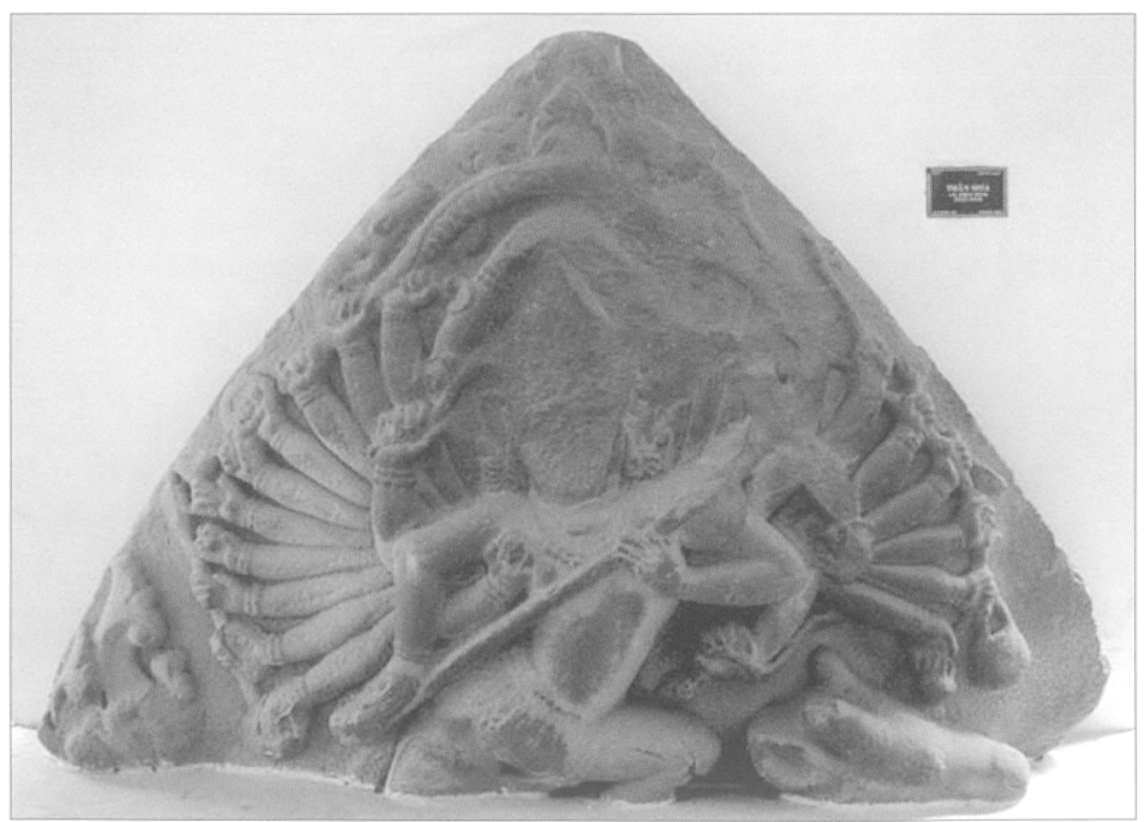

Ill. 6 : Śiva dansant, Khương $M y ̃, x^{e}$ siècle, musée de sculpture cam de Đà Nã̃ng (numéro d'inventaire « $15.7 »$; hauteur : $152 \mathrm{~cm}$; largeur : $107 \mathrm{~cm}$ ) (cliché : Pierre Baptiste / musée Guimet). 


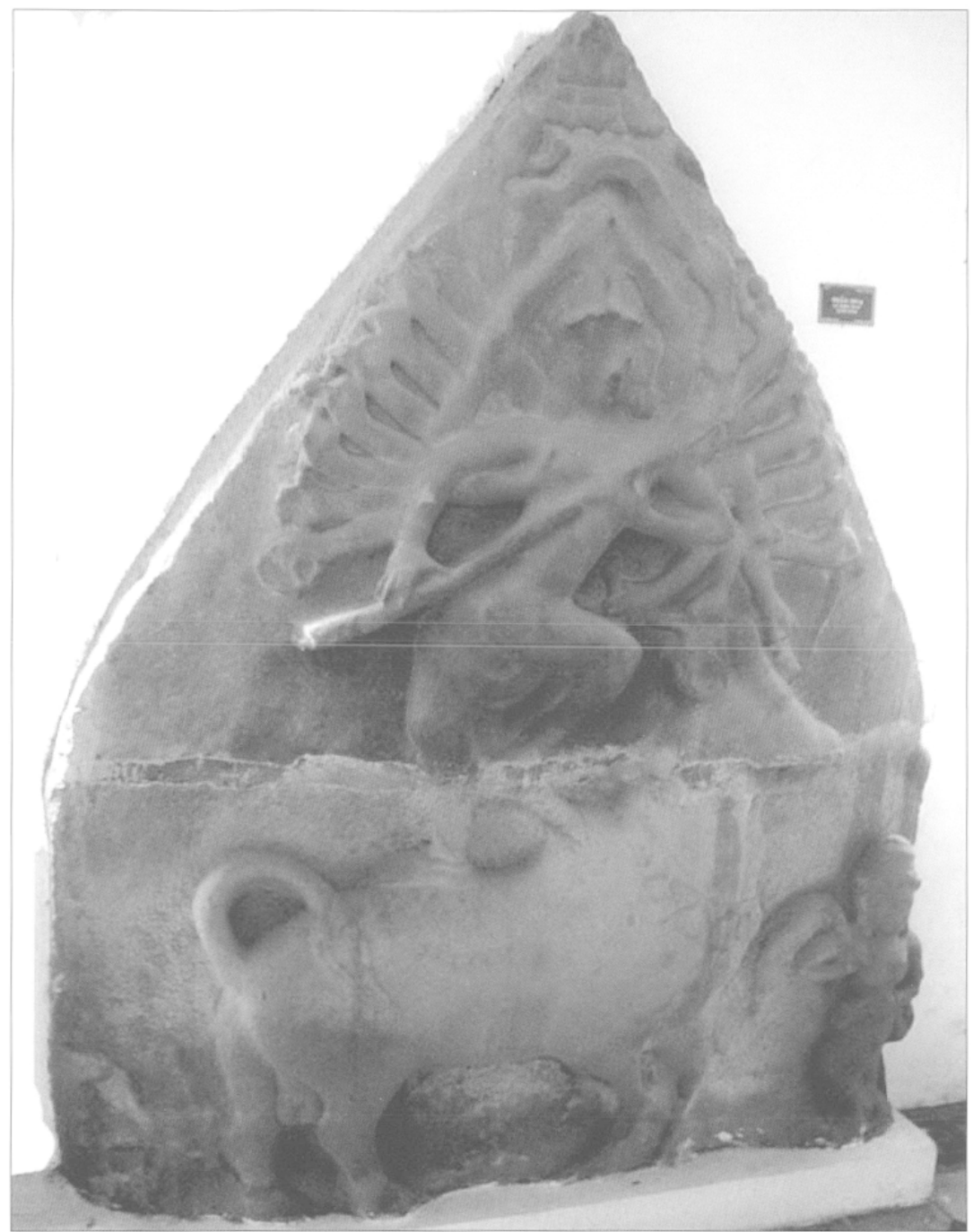

III. 7 : Śiva dansant, Khương Mỹ, $x^{e}$ siècle, musée de sculpture cam de Đà Nẵng (numéro d'inventaire « $15.6 »$; hauteur : $208 \mathrm{~cm}$; largeur : $159 \mathrm{~cm}$ ) (cliché : Pierre Baptiste / musée Guimet). 


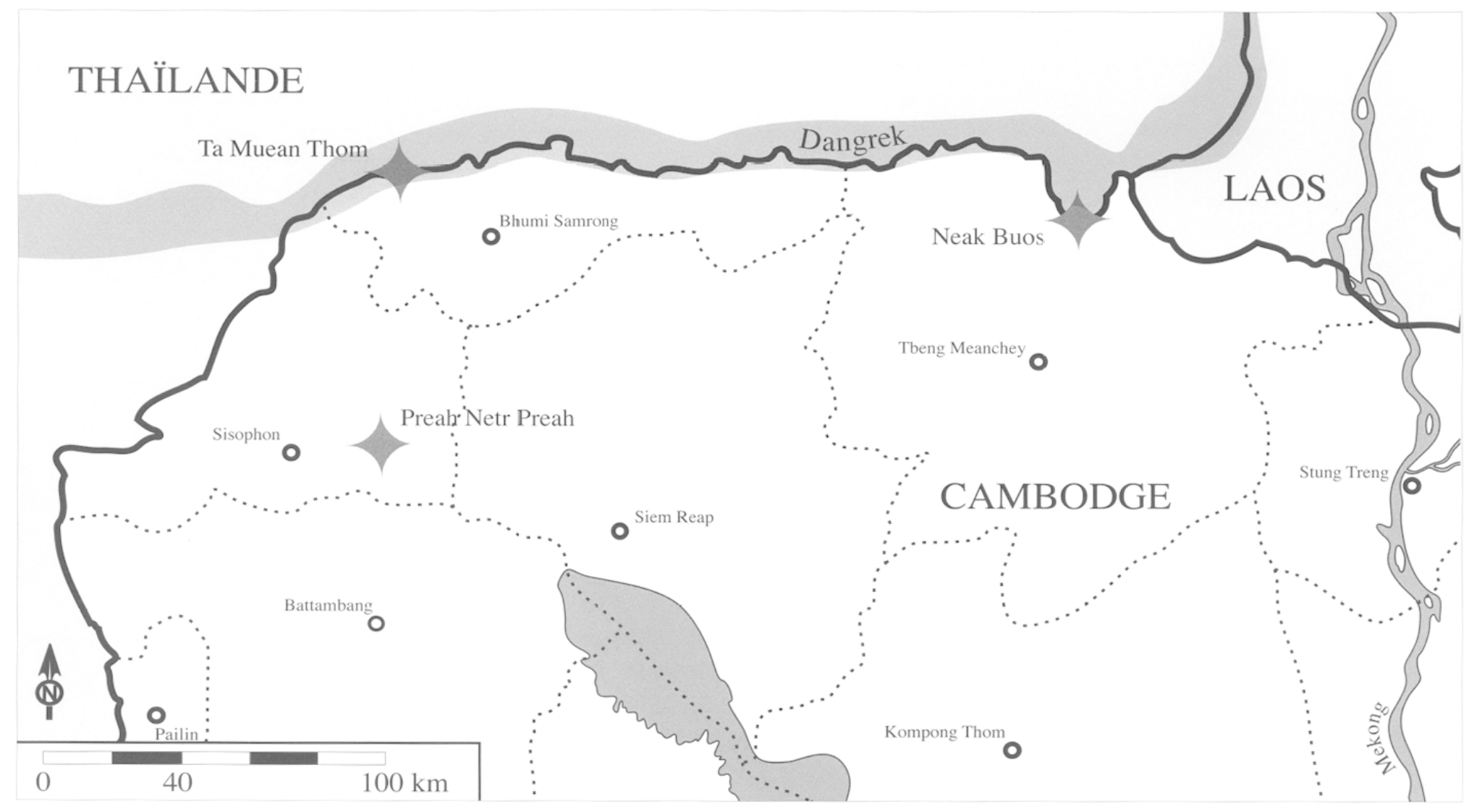

III. 1. Localisation des Śivapāda (d'après É. Lunet de Lajonquière, Inventaire descriptif des monuments du Cambodge, Cartes, Paris, 1911).

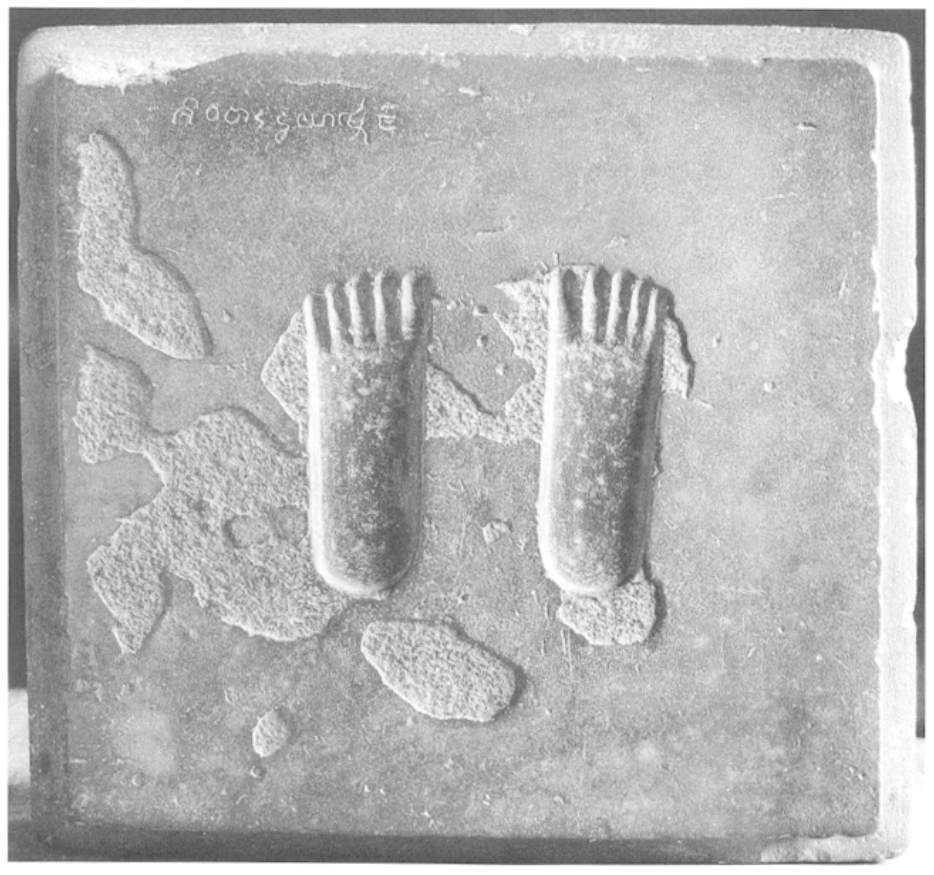

III. 2. " Pieds de Śiva » portant l'inscription K. 474 ; grès ; $32 \times 32 \times 16 \mathrm{~cm}$ (dont 8 pour le tenon); musée national du Cambodge, Phnom Penh ( $k a 1756$; cliché EFEO AMPP 942). 